\title{
THE STRUCTURE AND METALLICITY GRADIENT IN THE EXTREME OUTER DISK OF NGC 7793
}

\author{
M. Vlajić ${ }^{1,2}$, J. Bland-Hawthorn ${ }^{2}$, and K. C. Freeman ${ }^{3}$ \\ ${ }^{1}$ Astrophysikalisches Institut Potsdam, An der Sterwarte 16, D-14482 Potsdam, Germany \\ ${ }^{2}$ Sydney Institute for Astronomy, School of Physics, University of Sydney, NSW 2006, Australia; vlajic@aip.de \\ ${ }^{3}$ Mount Stromlo Observatory, Private Bag, Woden, ACT 2611, Australia \\ Received 2009 October 8; accepted 2010 December 21; published 2011 April 7
}

\begin{abstract}
Studies of outer regions of spirals disks are fundamental to our understanding of both the process of galaxy assembly and the subsequent secular evolution of galaxies. In an earlier series of papers, we explored the extent and abundance gradient in the outer disk of NGC 300 and found an extended purely exponential disk with a metallicity gradient which flattens off in the outermost regions. We now continue the study of outskirts of pure disk spirals with another Sculptor Group spiral, NGC 7793. Using the Gemini Multi Object Spectrograph camera at Gemini South, we trace the disk of NGC 7793 with star counts out to $\sim 9$ scale lengths, corresponding to $11.5 \mathrm{kpc}$ at our calibrated distance of $3.61 \pm 0.53 \mathrm{Mpc}$. The outer disk of NGC 7793 shows no evidence of a break in its light profile down to an effective surface brightness of $\sim 30 \mathrm{mag} \operatorname{arcsec}^{-2}\left(\sim 3 \mathrm{mag} \mathrm{arcsec}^{-2}\right.$ deeper than what has been achieved with surface photometry) and exhibits a non-negative abundance gradient within the radial extent of our data.
\end{abstract}

Key words: galaxies: abundances - galaxies: individual (NGC 7793) - galaxies: stellar content - galaxies: structure

\section{INTRODUCTION}

The study of the extreme outer disks of nearby spirals has produced some remarkable findings in recent years. These advances have been brought together by a confluence of observational and theoretical discoveries. On the observational side, star counts offer a superior method for tracing faint stellar populations in the outskirts of galaxies, compared to traditional surface photometry (Pritchet \& van den Bergh 1994; Ferguson et al. 2002; Bland-Hawthorn et al. 2005; Irwin et al. 2005; de Jong et al. 2007; Mouhcine et al. 2010; Radburn-Smith et al. 2011). While surface photometry is limited by diffuse background sources at the level of $\sim 27 \mathrm{mag} \operatorname{arcsec}^{-2}$, observations of resolved stellar populations do not suffer from the same limitations and allow us to reach 3-5 mag $\operatorname{arcsec}^{-2}$ deeper in effective surface brightness. On the other hand, the $\mathrm{N}$-body/smoothed particle hydrodynamics and semi-analytical models of disk formation are now able to address the importance of secular effects in the evolution of spirals. Scattering of stars by transient spiral arms first studied by Sellwood \& Binney (2002) could potentially have a significant effect on the evolution of spiral disks (Roškar et al. 2008a, 2008b; Schönrich \& Binney 2009; Sánchez-Blázquez et al. 2009; Martínez-Serrano et al. 2009; Minchev \& Famaey 2010; Minchev et al. 2011). The effects of the secular evolution of galaxies are expected to be most prominent in the outermost parts of disks (Roškar et al. 2008b). Additionally, due to their long dynamical timescales, the outer regions of spirals have largely retained fossil record from the epoch of galaxy assembly in the form of spatial distributions, kinematics, ages, and metallicities of their stars. These factors make outskirts of spirals particularly useful for testing models of galaxy formation and evolution.

Predictions that models of secular evolution of spirals make for stellar ages and metallicities in disk outskirts (Roškar et al. 2008a, 2008b; Sánchez-Blázquez et al. 2009) are still to be confirmed in a sufficiently large sample of galaxies. Age behavior is very difficult to observe in galaxies too distant for the full star formation history to be modeled. Barker et al. (2007) and
Williams et al. (2009) demonstrate nicely how an age gradient can be derived over the whole disk of M33; however, this kind of work is only possible for the few closest spirals. On the other hand, abundance gradients in galaxies can be studied relatively easily out to a few Mpc using broadband photometry. It has been well established that in a disk which is growing inside out, metallicity decreases from the center of the galaxy outward (Goetz \& Koeppen 1992; Matteucci \& Francois 1989). However, an increasing number of galaxies show signs of an abundance gradient flattening in their outermost regions (Andrievsky et al. 2004; Yong et al. 2006; Carraro et al. 2007; Pedicelli et al. 2009; Bresolin et al. 2009b; Vlajić et al. 2009). While models mentioned above offer possible explanations for the flattening, its exact cause is not yet clear and abundance data on more galaxies of different sizes and outer disk structure will help explain the slope of abundance gradient in the outermost regions of spirals.

As discussed in our earlier papers, the Sculptor Group is an ideal laboratory for studies of pure spirals: (1) its high galactic latitude minimizes foreground extinction and contamination from Milky Way stars, (2) most of its galaxies are isolated disk systems, and (3) the distance of the Sculptor Group (2-4 Mpc) allows for the resolved stellar population studies from the ground. At the far side of the Sculptor Group $(\sim 3.6 \mathrm{Mpc})$, NGC 7793 is a late-type spiral with an absolute magnitude $M=-18.46$, slightly brighter than NGC $300(-18.12$; absolute magnitudes were derived using corrected total apparent magnitude from Carignan 1985 and the distance derived in Section 3.1). Carignan (1985) studied the light profile of NGC 7793 out to $6^{\prime}$ using surface photometry and derived a scale length of $1.10(1.16 \mathrm{kpc})$ in $B$. A neutral hydrogen study of Carignan \& Puche (1990) reveals an H I disk extending out to $11.2(11.8 \mathrm{kpc})$, with a surface density at the last data point of $\sim 0.01 M_{\odot} \mathrm{pc}^{-2}$.

The plan of the paper is as follows. In Section 2, we describe the observations, data reduction, photometry, and completeness analysis. Next, we present results for the distance of NGC 7793 as estimated from the tip of the red giant branch (TRGB; Section 3.1), color-magnitude diagram (CMD; Section 3.2), 


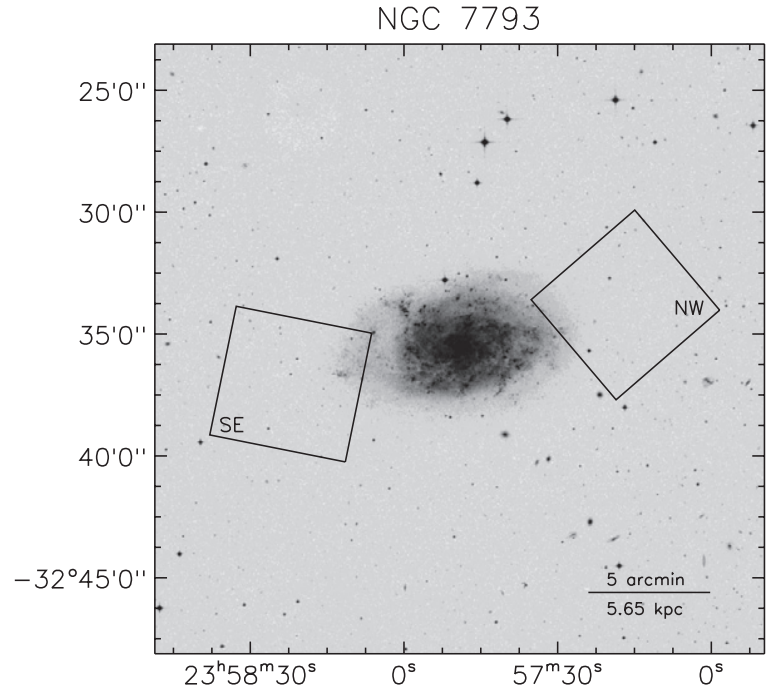

Figure 1. DSS wide-field image of NGC 7793 with GMOS fields overlaid.

star count profiles (Section 3.3), surface brightness profile (Section 3.4), and metallicity gradient (Section 3.5). Discussion and conclusions follow in Section 4.

\section{OBSERVATIONS, DATA REDUCTION, AND PHOTOMETRY}

\subsection{Observations and Data Reduction}

The data were obtained using the Gemini Multi Object Spectrograph (GMOS) on the Gemini South telescope over three nights in 2005 August as a part of the program GS-2005B-Q-4. Deep $g^{\prime}$ and $i^{\prime}$ images of two major axis fields on each side of the galaxy were taken; the locations of the fields (SE and NW) are shown in Figure 1. GMOS field of view is 5.5 on a side. (At the distance of NGC 7793 (3.61 Mpc, Section 3.1), 1' corresponds to $1.05 \mathrm{kpc}$.) The average FWHM of the data is $0{ }^{\prime \prime} 6$ (SE) and $0.8(\mathrm{NW})$ in the $g^{\prime}$, and $0{ }^{\prime} .5(\mathrm{SE})$ and $0.7(\mathrm{NW})$ in the $i^{\prime}$ band.

To reduce the data we employed the standard IRAF/Gemini routines which included (1) bias subtraction and flat fielding (gireduce), (2) (for $i^{\prime}$ data only) creating the master fringe frame from the individual reduced frames (gifringe) and subtracting it from the individual images (girmfringe), (3) mosaicking of individual GMOS CCDs into a single reference frame (gmosaic), and (4) combining the dithered exposures into a final image (imcoadd).

We obtained $13 \times 600 \mathrm{~s}$ exposures in $g^{\prime}$ and $22 \times 600 \mathrm{~s}$ exposures in the $i^{\prime}$ band per field, bringing the total on-source exposure time to $11.7 \mathrm{hr}$. The data for the SE field were taken during the nights of 2005 August 09 UT (hereafter first night) and 2005 August 10 UT (hereafter second night). Only 5 (out of 13) $g^{\prime}$-band and 21 (out of 22) $i^{\prime}$-band images were observed on the first night and the observing log indicated that a thin cloud might have affected $i^{\prime}$-band observations. In addition, only one standard star field observation was recorded. Remaining science images of SE field, as well as three standard stars fields, were observed the following night. The bulk of the data of the NW field was collected on the night of 2005 August 11 UT (12 $g^{\prime}$ band and $21 i^{\prime}$-band images, hereafter third night). However, no photometric standard stars were observed that night. The remaining science frames (one in each of the bands) were observed the previous night under photometric conditions.

Initial analysis of the photometry revealed a suspicious discrepancy in $i^{\prime}$-band magnitude distribution between the two fields. In correspondence with the Gemini staff it was confirmed that this was most likely due to the non-photometric conditions on the night of 2005 August 09 UT, when the majority of the science frames of the SE field were taken. Accounting for this, and the fact that the photometric standard stars observation were only taken on the second night of the run, we decided to proceed in the following manner.

1. SE field, $i^{\prime}$ band: combine 21 images taken on the first night and compare photometry of this deep image with the photometry extracted from the single image observed on the second night.

2. SE field, $g^{\prime}$ band: combine separately five images observed on the first, and eight images taken on the second night and compare the photometry between the two images.

3. NW field, $i^{\prime}$ band: compare the photometry extracted from the deep image created by combining 21 images taken on the third night of the run with the single image taken on the second night.

4. NW field, $g^{\prime}$ band: similarly to the $i^{\prime}$-band case, compare a combined deep image created from the 12 exposures taken on the third night, with the single image taken on the second night of the run.

In the following sections, we will refer to the (combinations of) images taken on the second night of the run as calibration images and to those observed on the first and third night as final images.

\subsection{Photometry and Artificial Star Tests}

To extract stellar photometry we used the standalone version of DAOPHOT and ALLSTAR packages (Stetson 1987). Following the initial runs of FIND and PHOTOMETRY routines, which were used to catalog objects in the image and measure their aperture photometry, we proceeded to determine the pointspread function (PSF) for each image. Depending on the filter and the field, 80-220 moderately bright isolated stars in each field were selected as PSF stars and used to iteratively compute the PSF. The PSF stars were "hand-picked" and their radial, contour, and mesh profiles were visually examined within the IRAF/DAOPHOT package. The calculated PSF was used to subtract the PSF stars from the original image; the positions of the subtracted PSF stars were inspected again and the PSF stars which did not subtract cleanly were excluded from the PSF calculation. In addition, stars with subtraction errors which differed more than $3 \sigma$ from the mean value were also excluded. The next iteration of the PSF was calculated using images in which, within the fitting radius of each PSF star, all but PSF stars have been subtracted. This was followed with yet another visual inspection within IRAF/DAOPHOT as described above. The whole procedure was repeated once more to derive the final PSF. Finally, ALLSTAR was used to fit the calculated PSF to all stars in the object catalogs and determine their photometry.

Comparison of the final and calibration images revealed the following. In the $i^{\prime}$ band, we found the difference in photometry extracted from the calibrated and final images of $0.396 \pm 0.012$ for the SE field and $0.073 \pm 0.006$ for the NW field. In the $g^{\prime}$ band, we found, within uncertainties $(2 \sigma)$, no difference in magnitudes between calibration and final images. We therefore decided, in the subsequent analysis, to use the master frames created by combining the full sample of 13 frames in $g^{\prime}$, and a reduced sample of 21 images in the $i^{\prime}$ band. Two $i^{\prime}$-band images, one of each field, taken on the second night were used to calibrate the photometry of the final images which were very likely taken under non-photometric conditions. 
We performed photometric calibration using the standard stars observations taken during the second night of the observing run. The derived zero points were in excellent agreement with those stated on the Gemini Web site (28.31 (our value) versus 28.33 (GMOS value) for $g^{\prime}$ and 27.92 (our value) versus 27.93 (GMOS value) for the $i^{\prime}$ band). In addition, we applied additional offset (0.396 for the SE field, 0.073 for the NW field) to the photometry derived from our final $i^{\prime}$ images, as explained above.

The DAOGROW software suite (Stetson 1990) was used to calculate aperture corrections. We subtracted from each image all but PSF stars and determined their aperture photometry in a series of apertures. DAOGROW uses the information on aperture photometry to derive the "total" magnitude of a star. The weighted mean difference between the PSF-based magnitude (from ALLSTAR) and the "total" magnitude (from DAOGROW) of the PSF stars was adopted as "aperture correction" and applied to PSF-based magnitudes of all stars in the frame. The uncertainties in the aperture correction were in the range $0.006-0.008$.

Completeness, crowding, and photometric uncertainties of the data were assessed using artificial star tests, described in more detail in Vlajić et al. (2009). We added 900 artificial stars to each frame in a $30 \times 30$ grid with the cell size of 69 pixels $\left(10^{\prime \prime}\right)$; for each combination of field and filter 100 artificial star test runs were performed with the grid origin randomly offset between the runs. Photometry (i.e., magnitude range and color distribution) of artificial stars was determined by randomly sampling the original CMDs. Artificial stars were added to the frames using the DAOPHOT/ADDSTAR routine and the resulting images were analyzed with the identical data reduction pipeline as the original frames. We consider an artificial star recovered if it is detected in both filters with the difference between input and recovered magnitude $<0.5$ mag. Completeness as function of magnitude is calculated as a ratio between the number of recovered and input stars in a given magnitude bin. Our photometry is $50 \%$ complete down to $\left(g^{\prime}, i^{\prime}\right)=(27.35-27.47,26.75-27.10)$. We also calculate radial completeness as a ratio of the number of recovered and input stars in a given radial bin; the information is later used to correct radial star counts and effective surface brightness profiles for crowding in the innermost regions. Furthermore, we used information on the $\mathrm{CHI}$ and SHARP parameters of recovered artificial star tests to discard spurious objects from the ALLSTAR catalog. CHI and SHARP are often employed as indicators of the non-stellar nature of an object; outliers in the plots of CHI and SHARP as a function of magnitude can be relatively safely assumed to be semi-resolved galaxies, blends, cosmic rays, or image defects. We removed from further analysis all stars lying outside the envelope demarcating three standard deviations from the artificial stars' mean CHI and SHARP values. These cuts, together with a cut on error in color $(\approx 0.15)$, remove $\sim 50 \%$ of objects from the original matched catalogs.

\section{RESULTS}

\subsection{Tip of the Red Giant Branch Distance}

Stellar evolution models (e.g., Salaris \& Cassisi 1997; Iben \& Renzini 1983) show that below a metallicity of $[\mathrm{Fe} / \mathrm{H}] \approx-0.7$ the brightness of the TRGB is expected to be roughly constant, regardless of stellar age and metallicity. Observationally, Da Costa \& Armandroff (1990) and Bellazzini et al. (2001) confirmed that for a sample of Galactic globular clusters spanning

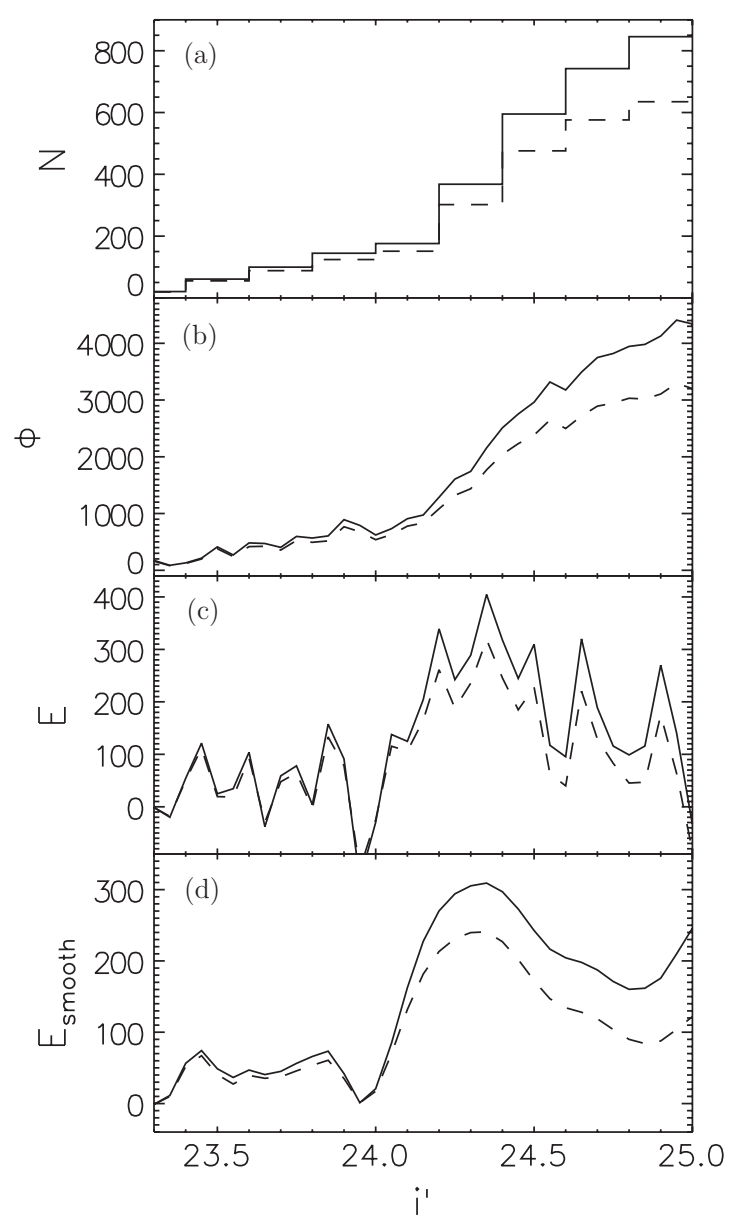

Figure 2. Determination of the TRGB. $i^{\prime}$-band luminosity function (a) and smoothed luminosity function (b) are shown in the top panels before and after completeness correction (dashed and solid lines, respectively). Outputs of the edge-detection filter are shown in the bottom two panels, calculated using the photometric error as determined from artificial star tests (c), and calculated using a photometric error four times larger than calculated from artificial star tests (d); line types are the same as in the top panels.

a large range of abundances $(-2.1<[\mathrm{Fe} / \mathrm{H}]<-0.7)$ and ages (2-15 Gyr), the absolute $I$-band magnitude of the TRGB is stable and insensitive to age and metallicity, which enables its application as an extragalactic distance estimator. In general, there has been a good agreement between the distances obtained using the TRGB and classical methods such as Cepheids (Sakai et al. 1996; Ferrarese et al. 2000).

Following Lee et al. (1993) and Sakai et al. (1996), we employ our deep $i^{\prime}$-band photometry of two fields in the outskirts of NGC 7793 to determine the galaxy's distance. Figure 2 shows the result of this exercise. In order to increase the sample of stars used in the detection, we combined photometry of the two fields. This results in a value consistent with the one we derive from each field separately, while having a narrower and better defined peak.

The top panel of the figure shows the $i^{\prime}$-band luminosity function, with (solid line) and without (dashed line) the completeness correction. In correcting for incompleteness, we only consider stars that are above $50 \%$ completeness limit in both $g^{\prime}$ and $i^{\prime}$. In the middle panel, the smoothed luminosity function is shown. In calculating this function, discrete stellar magnitudes are replaced by Gaussians with the width equal to the star's photometric error, according to the expression (Sakai et al. 1996) 


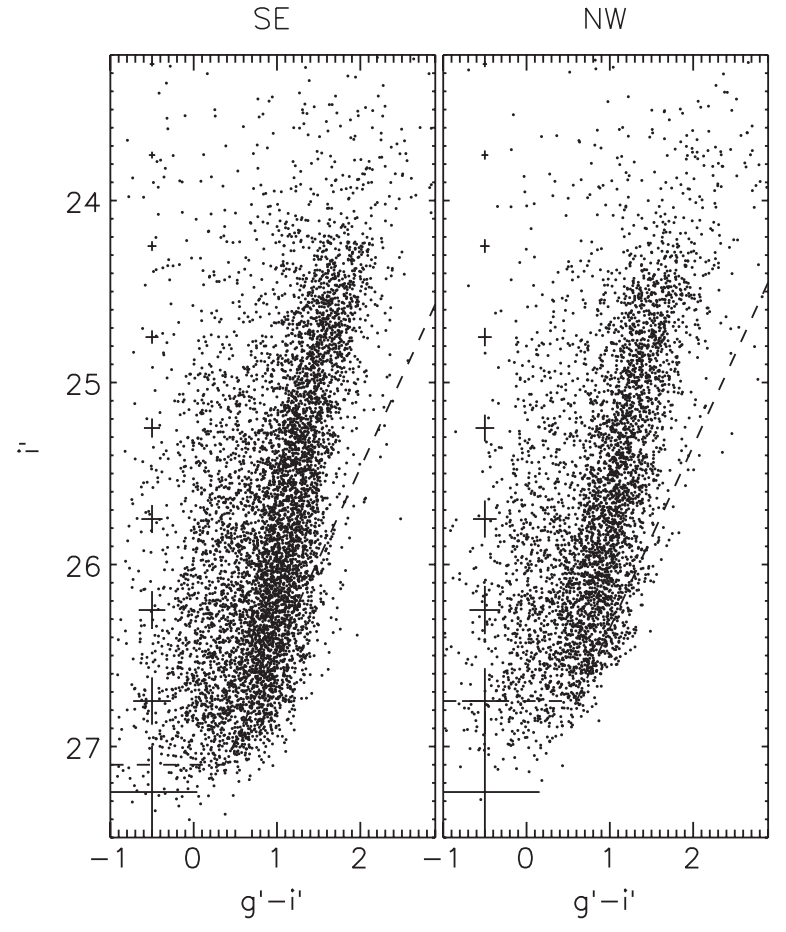

Figure 3. CMD of two NGC 7793 fields. Dashed lines represent the 50\% completeness limit. Photometric uncertainties at a given magnitude and color of $g^{\prime}-i^{\prime}=1$ are shown on the left of the panels.

$$
\Phi(m)=\sum_{i=1}^{N} \frac{1}{\sqrt{2 \pi} \sigma_{i}} \exp \left[-\frac{\left(m_{i}-m\right)^{2}}{2 \sigma_{i}^{2}}\right]
$$

A smooth adaptive edge-detection filter of the form

$$
E(m)=\Phi\left(m+\bar{\sigma}_{m}\right)-\Phi\left(m-\bar{\sigma}_{m}\right)
$$

is then applied. Here, $\bar{\sigma}_{m}$ is the mean photometric error of all stars in the magnitude range $[m-0.05, m+0.05]$, as determined from artificial star tests. The maximum of the output of this edge-detection filter (shown in Figure 2(c)) marks the TRGB. Relatively small photometric errors cause the output of the edge-detection filter to be noisy. In order to obtain a smoother output which would yield a more reliable distance estimate, we calculate the same edge-detection function using photometric errors three times those estimated from the artificial star tests. The result is shown in the bottom panel of Figure 2. The apparent $i^{\prime}$ magnitude of the TRGB is estimated to be $i_{\text {TRGB }}^{\prime}=24.35 \pm 0.30$. Uncertainties are determined as halfwidth at half-maximum of the peak in Figure 2(d). With the absolute $i^{\prime}$-band magnitude $M_{i^{\prime}, \mathrm{TRGB}}=-3.44 \pm 0.10$ (Bellazzini 2008), we calculate the NGC 7793 distance modulus to be $m-M=27.79 \pm 0.32$, corresponding to the distance of $3.61 \pm 0.53 \mathrm{Mpc}$. This is in good agreement with the values obtained by Karachentsev et al. $(2003 ; 3.91 \pm 0.41 \mathrm{Mpc})$ and the GHOSTS survey (3.73 Mpc; Radburn-Smith et al. 2011).

\subsection{Color-Magnitude Diagram}

CMDs of two NGC 7793 fields are shown in Figure 3. Also marked in the figure are 50\% completeness limit and photometric errors as determined from artificial star tests at a given $i^{\prime}$ magnitude and color of $g^{\prime}-i^{\prime}=1$. The $\left(g^{\prime}-i^{\prime}, i^{\prime}\right)$ CMDs reveal a prominent red giant branch (RGB). RGB stars make up more than $60 \%$ of detections in each field. Region just

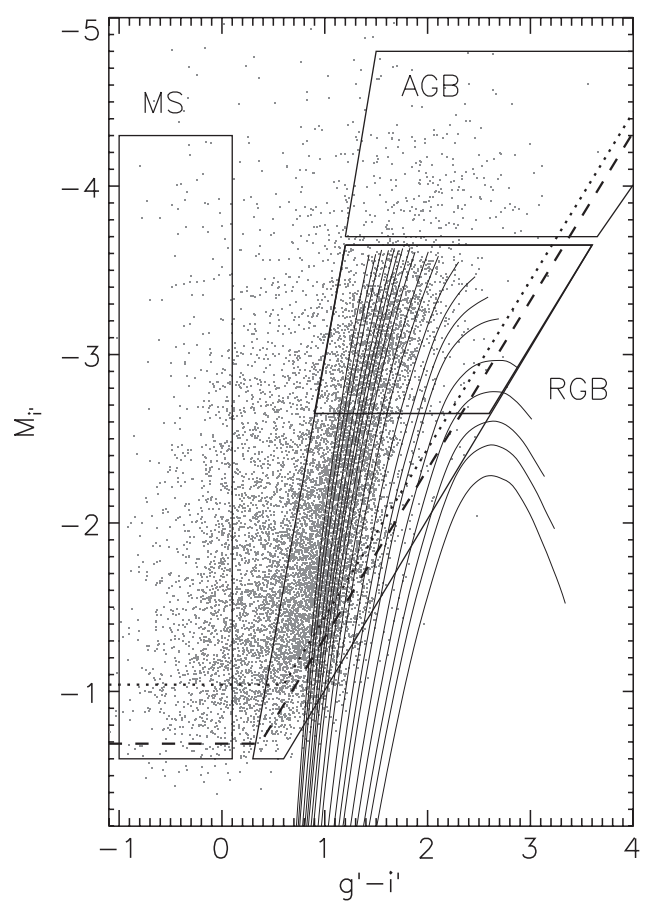

Figure 4. CMD of both NGC 7793 fields with selection boxes for RGB, AGB, and MS populations marked. Dashed lines represent the 50\% completeness limit for the SE field (dashed line) and the NW field (dotted line).

blueward of the RGB is most likely populated by unresolved faint background galaxies. Also potentially visible in the CMD is a main sequence (MS) and an asymptotic giant branch (AGB) population. Selection boxes used for deriving properties of individual stellar populations are shown in Figure 4. AGB and MS populations make up $\sim 2 \%$ and $\sim 10 \%$ of all objects in the field.

\subsection{Star Count Profiles}

We derive radial profiles of NGC 7793 in order to examine the extent and structure of its outer disk. Star counts profiles are computed by counting up the stars in elliptical annuli with inclination and position angle corresponding to that of NGC 7793. These raw counts are then normalized by the area sampled by each annulus.

The most challenging aspect of studying outer disk light profiles is the need to determine a background level that is then subtracted from the raw profile in order to calculate the true star counts or surface brightness profile of a galaxy. In the case of surface photometry, this is manifested through difficulties in deriving accurate estimates of sky brightness. When resolved stellar photometry is used instead to study outskirts of spirals, the problem translates into how to reliably evaluate the number of unresolved faint background galaxies that are mistakenly included into stellar catalogs. Unlike in the case of surface photometry, in star counts studies we are not limited by the fundamental limits of observations but by the accuracy with which we can determine the galaxy number counts. However, the challenge is particularly difficult when information on number counts as a function of color (and not only magnitude) is required. We therefore pay careful attention to estimating the number counts of faint background galaxies.

In Figures 5(a) and (e), we show star count profiles constructed using all stars in the field, irrespective of their colors. We use the GalaxyCount online tool (Ellis \& Bland-Hawthorn 


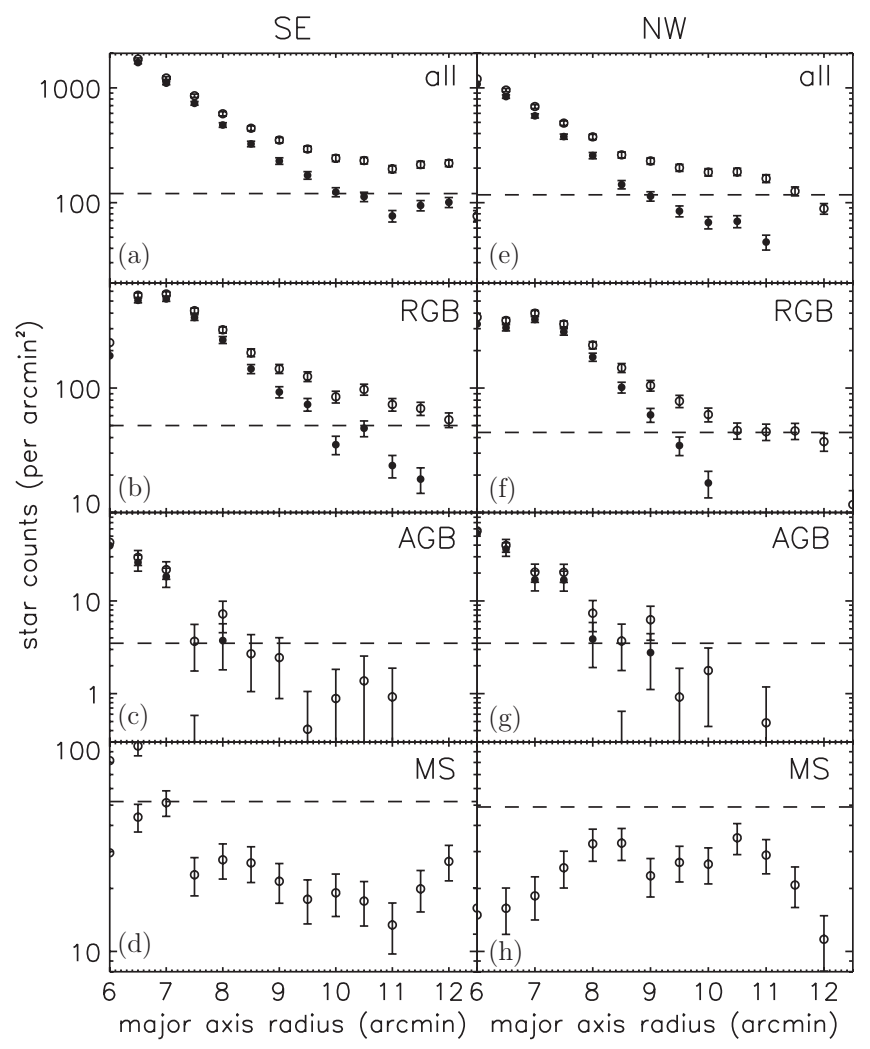

Figure 5. Star counts profiles of the SE field (left panels) and NW field (right panels). From top to bottom, the profiles shown are of all stars (a and e), RGB (b and f), AGB (c and g), and MS stars ( $\mathrm{d}$ and $\mathrm{h}$ ). In each panel, the dashed line displays the estimated galaxy counts level, determined using methods described in the text. Open and full symbols show the star counts before and after the background galaxy contribution has been subtracted.

2007) to determine galaxy number counts in the magnitude range probed by our data; dashed horizontal line in top panels shows the estimated background galaxy level of $120( \pm 12)$ $\operatorname{arcmin}^{-2}$ (SE) and 117( \pm 12$) \operatorname{arcmin}^{-2}$ (NW field). The open and full symbols in the figure represent the star counts before and after the number counts of galaxy contaminants have been subtracted. Figure 5(e) shows that the star counts profile of the NGC 7793 NW field falls off to practically zero within the extent of our data, at the radius of approximately $11^{\prime}$ or $11.5 \mathrm{kpc}$. It is unclear whether the upturn reflected in the two outermost data points for the SE field (Figure 5(a)) is real, or (possibly more likely) a result of a slight underestimation of background counts.

We also calculate the star counts profiles of distinct stellar populations in the outer disk of NGC 7793 (Figures 5(b)-(d) and (f)-(h)). Selection boxes used to separate stars into different populations are marked on the CMD in Figure 4. Since the GalaxyCount tool does not provide direct information on the color of background galaxies, we require an alternative method for determining the galaxy number counts. Following Vlajić et al. (2009), we employ the data from the William Herschel Deep Field (WHDF; Metcalfe et al. 2001) to estimate the contamination from the faint background galaxy population. We determine the number counts within the AGB box directly from the WHDF data, while for the stellar populations reaching fainter magnitudes than probed with WHDF (i.e., MS and RGB stars) we use the method described in Vlajić et al. (2009) to calculate the galaxy number counts. We calculate the $i^{\prime}$-band number counts of all galaxies in WHDF and fit linearly the (log of) differential number counts in $0.5 \mathrm{mag}$ bins. In order to determine the galaxy counts below the limit of the WHDF survey we assume that the counts in the bins 2-3 mag below the survey limit follow the same linear trend (in the log space) as the counts in the brighter bins used in the fit. We finally correct the derived galaxy number counts using completeness curves of our data. The resulting background galaxy counts are $3.9 \pm 2.1,51 \pm 7(44 \pm 7)$ and $52 \pm 8(49 \pm 7) \mathrm{arcmin}^{-2}$, for the AGB, RGB, and MS selection regions, respectively, for the SE (NW) field. (Quoted errors are variance, as estimated by GalaxyCount.) While contamination-subtracted profiles of RGB stars largely confirm the finding from Figures 5(a) and (e) (with the distinction that the RGB profile for the NW field falls off more steeply and RGB stars are only detected out to $10^{\prime}$ ) we detect no MS stars and all objects within our MS selection box can be attributed to the contaminating background galaxy population (the galaxy number counts for the MS selection box are $\sim 2-3$ times higher than the derived star counts for this CMD). We detect AGB stars out to 8-9' (8.5-9.5 kpc), after which their number counts fall below the estimated background galaxy level. As we show in Vlajić et al. (2009), at the high galactic latitudes of the Sculptor Group, contamination from the Milky Way stars is negligible (Robin et al. 2003; Sharma et al. 2011).

D. J. Radburn-Smith (2011, in preparation) finds a break in the radial profile of young and intermediate age stars in the outer disk of NGC 7793 (their Hubble Space Telescope/Advanced Camera for Surveys fields overlap significantly with our SE field), with the scale length of a stellar population being shorter for younger stars. This is largely consistent with the star count profiles we derive. Due to the higher level of contamination in our ground-based data we see no MS stars, in agreement with the short scale length for this population found by D. J. RadburnSmith (2011, in preparation); similarly, we find AGB stars to be more extended than the MS population, with the RGB stars having the largest scale length.

Comparing the RGB profiles of NGC 7793 and NGC 300 (Vlajić et al. 2009, Figure 9) we find that the counts in the outermost bins shown in Figure 5(b) are $\sim 2$ times lower than corresponding counts in the most distant bins in the outer disk of NGC 300. This is yet another piece of evidence supporting our earlier finding of an extended exponential disk in NGC 300 (Bland-Hawthorn et al. 2005; Vlajić et al. 2009). While our CMD of NGC 300 reaches 4 mag below the tip of the RGB, compared to only $2.5 \mathrm{mag}$ in NGC 7793, background galaxy number counts increase rapidly with magnitude and the counts in the faintest magnitude bins dominate the total galaxy counts. Our CMDs of NGC 300 and NGC 7793 reach same apparent depth ( $26.5-27 \mathrm{mag})$ and hence experience roughly the same contamination by faint background galaxies. The difference in star counts in the outermost bins therefore does not reflect the difference in galaxy number counts but in star counts, and is an additional independent confirmation of the extended exponential disk in NGC 300 out to at least 10 disk scale lengths.

\subsection{Surface Brightness Profile}

The power of resolved stellar photometry over surface photometry is most easily recognized if star counts are transformed into measurements of effective surface brightness and compared with existing surface brightness data. It has been shown in a number of works recently (Bland-Hawthorn et al. 2005; Irwin et al. 2005; de Jong et al. 2007; Radburn-Smith et al. 2011) that this approach allows one to reach surface brightnesses 3-4 mag $\operatorname{arcsec}^{-2}$ below the limit of surface photometry. 

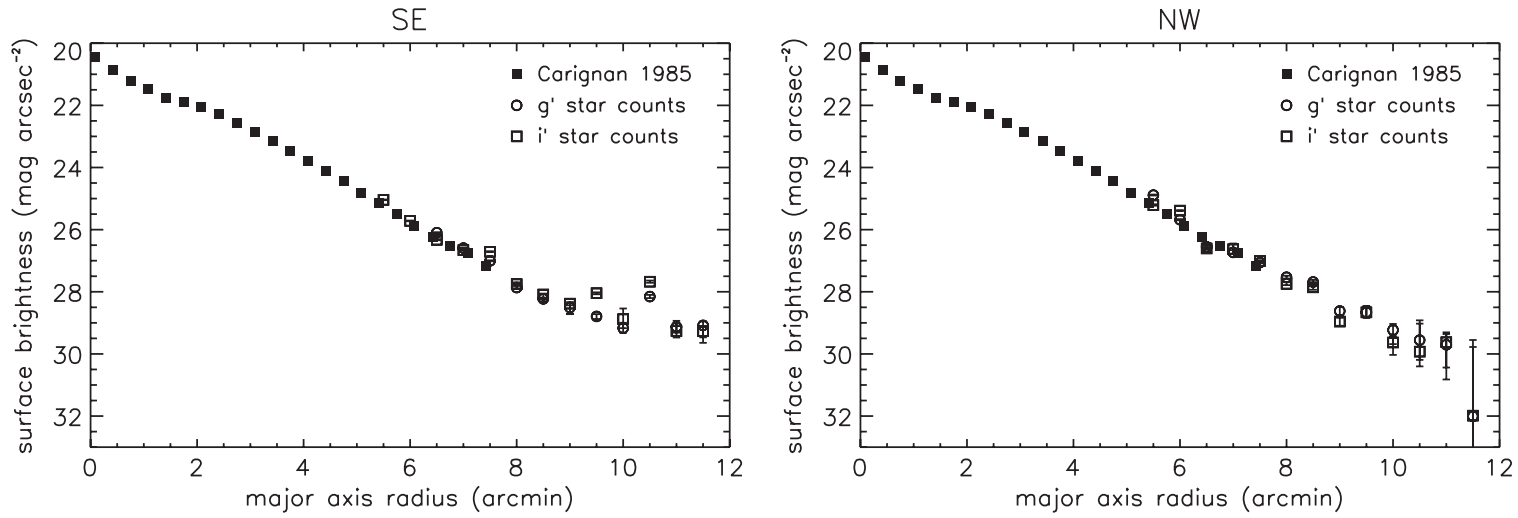

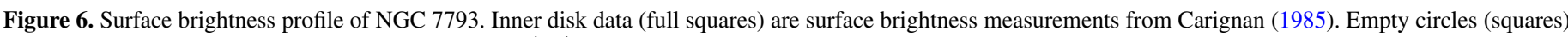

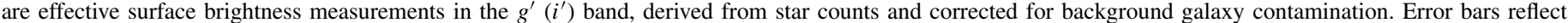

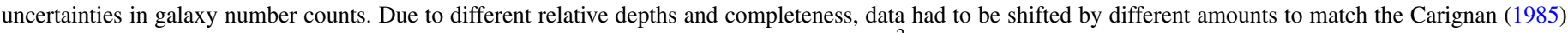

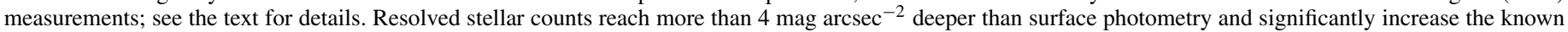
extent of NGC 7793.

We divide the data in 0.5 wide annuli and calculate surface brightness in each annulus as

$$
\mu=-2.5 \log \left(\frac{F^{\prime \prime}}{N_{\text {pix }}} f_{g^{\prime}} f_{i^{\prime}} \sum_{i} 10^{-0.4 m_{i}}\right) .
$$

Here, $N_{\text {pix }}$ is a number of pixels in an annulus, $f_{g^{\prime}}$ and $f_{i^{\prime}}$ are radial completeness factors, and $m_{i}$ are magnitudes of stars within a given annulus. Radial completeness of our data is lowest in the innermost annulus (45\%) due to crowding, and increases to an average of $87 \%$ in the outermost disk. To convert surface brightness to units of mag $\operatorname{arcsec}^{-2}$ we multiply the effective flux under the logarithm with the inverse of the square of the GMOS pixel size ( 1 pixel $\left.=00^{\prime \prime} 146\right)$ which is equal to $F^{\prime \prime}=1 / 0.146^{2}=47$.

In order to take into account the contribution from faint background galaxies (see Section 3.3) we estimate contamination in each annulus as

$$
N_{\text {cont }}=\frac{N_{\text {gal }} N_{\text {pix }}}{F^{\prime}}
$$

where $F^{\prime}=168887$ is a scale factor equivalent to $F^{\prime \prime}$ that converts $N_{\text {pix }}$ to $\operatorname{arcmin}^{2}$ and $N_{\text {gal }}=120( \pm 12) \operatorname{arcmin}^{-2}$ for SE and $117( \pm 12)$ for NW field (Section 3.3). We next perform 100 realizations of background subtraction; in each of these we remove a random set of $N_{\text {cont }}$ stars from each annulus and calculate surface brightness using the remaining stars as described above. The final surface brightness profile is the mean of a hundred realizations. We find the standard deviation of the whole set of realizations for a given annulus not to be significant $\left(<0.03 \mathrm{mag} \operatorname{arcsec}^{-2}\right.$ in $g^{\prime}$ and $<0.05 \mathrm{mag} \mathrm{arcsec}^{-2}$ in $i^{\prime}$ ), confirming that the derivation of surface brightness profile is robust against the choice of objects we exclude as contamination.

The derived $g^{\prime}$ and $i^{\prime}$ effective surface brightness profiles are shown in Figure 6. The inner disk data points in the figure are from the Carignan (1985) study. Since, to minimize spurious detections, we used the matched stellar catalog rather than individual $g^{\prime}$ and $i^{\prime}$ catalogs when deriving surface brightness, it is not surprising to find that $g^{\prime}$ and $i^{\prime}$ profiles are practically identical. The uncertainty in the galaxy number counts is reflected in the error bars in Figure 6. Low/high error bar limits represent surface brightness profiles calculated using
Table 1

NGC 7793 Disk Scale Length

\begin{tabular}{lccc}
\hline \hline Field & Filter & Scale Length $\left(^{\prime}\right)$ & Scale Length $(\mathrm{kpc})$ \\
\hline Carignan & $B_{J}$ & $1.11 \pm 0.01$ & $1.17 \pm 0.02$ \\
SE & $g^{\prime}$ & $1.12 \pm 0.05$ & $1.18 \pm 0.05$ \\
SE & $i^{\prime}$ & $1.24 \pm 0.09$ & $1.30 \pm 0.10$ \\
NW & $g^{\prime}$ & $1.12 \pm 0.07$ & $1.18 \pm 0.07$ \\
NW & $i^{\prime}$ & $1.05 \pm 0.06$ & $1.10 \pm 0.07$ \\
\hline
\end{tabular}

background galaxy number counts that are $1 \sigma$ lower/higher than number counts used to derive the original profile. In order to match the inner disk result, effective surface brightness data points of the NW field have been shifted downward by 1.3 and $0.3 \mathrm{mag}$ in $g^{\prime}$ and $i^{\prime}$, respectively. For the SE field, corresponding values are 0.9 and 0.0 in $g^{\prime}$ and $i^{\prime}$.

Fitting an exponential to a light profile in Figure 6 we calculate the scale length of the NGC 7793 disk. Carignan (1985) data points yield for the disk scale length a value of 1 '.11 \pm 0.02 $(1.17 \pm 0.02 \mathrm{kpc})$. The scale lengths derived using our data, excluding data points at 10.5 and $11^{\prime}$, are presented in Table 1 and are largely in agreement with the Carignan's value.

In summary, our effective surface brightness derived from star counts reaches $\sim 3 \mathrm{mag} \operatorname{arcsec}^{-2}$ deeper than the surface brightness profile of Carignan (1985). We trace the exponential light profile out to $\sim 9$ disk scale lengths, greatly increasing the known extent of the disk from the Carignan study. There is no detectable break in the surface brightness profile and the disk remains exponential out to $11^{\prime}(11.5 \mathrm{kpc})$.

\subsection{Metallicity Distribution Function and Metallicity Gradient in the Outer Disk}

Using the position of stars on the RGB as a proxy for their metallicities, we derive the metallicity distribution function (MDF) and the metallicity gradient of the stars in the outer disk of NGC 7793. Due to higher sensitivity of the color of RGB stars on metallicity than age (e.g., VandenBerg et al. 2006), the age-metallicity degeneracy does not have a significant effect on the shape of the derived MDF and metallicity gradient, and allows for a relatively accurate abundance determination from broadband photometry.

In calculating the metallicity gradient, we only use stars from the top $\sim 1$ mag of the RGB selection box in Figure 4 (i.e., 

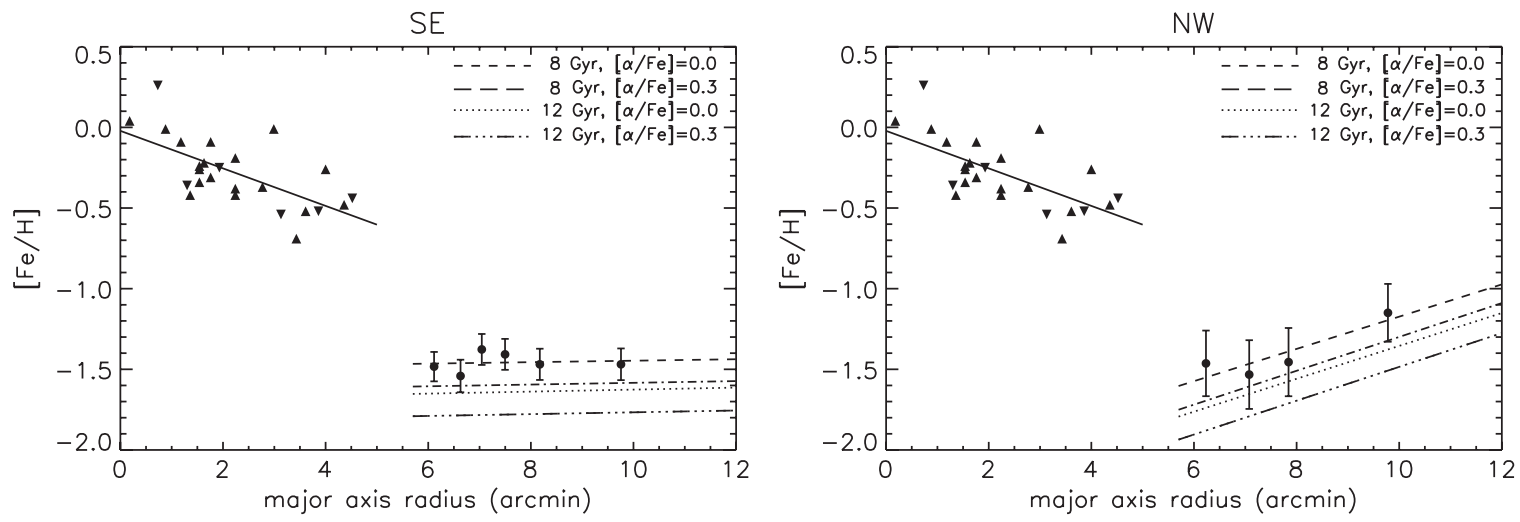

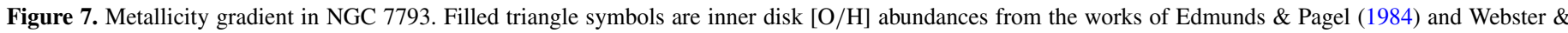

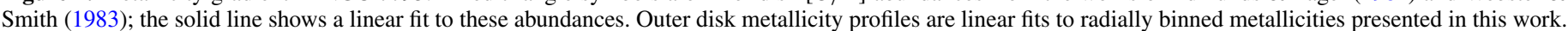

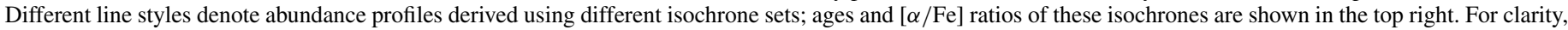

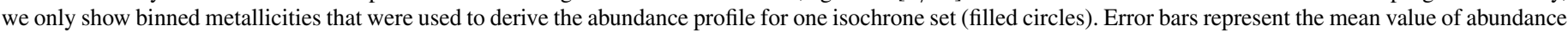
uncertainties in each bin.

Table 2

Metallicity Gradient in NGC 7793

\begin{tabular}{lcccccc}
\hline \hline Field & Age $(\mathrm{Gyr})$ & {$[\alpha / \mathrm{Fe}]$} & $a$ & $\sigma_{a}$ & $b$ & $\sigma_{b}$ \\
\hline SE & 8 & 0.0 & 0.004 & 0.011 & -1.49 & 0.10 \\
SE & 8 & 0.3 & 0.006 & 0.011 & -1.69 & 0.11 \\
SE & 12 & 0.0 & 0.005 & 0.012 & -1.64 & 0.11 \\
SE & 12 & 0.3 & 0.005 & 0.011 & -1.82 & 0.11 \\
NW & 8 & 0.0 & 0.095 & 0.023 & -2.17 & 0.20 \\
NW & 8 & 0.3 & 0.097 & 0.024 & -2.37 & 0.21 \\
NW & 12 & 0.0 & 0.100 & 0.023 & -2.35 & 0.21 \\
NW & 12 & 0.3 & 0.100 & 0.024 & -2.53 & 0.21 \\
\hline
\end{tabular}

Notes. Gradients are in the form of $[\alpha / \mathrm{Fe}]=a R+b$, where $a$ and the corresponding error have units of dex $\mathrm{kpc}^{-1}$. The abundance gradient for the inner disk using the data on $[\mathrm{O} / \mathrm{H}]$ abundances of $\mathrm{H}$ II regions from Edmunds \& Pagel (1984) and Webster \& Smith (1983) is $-0.11 \pm 0.02 \mathrm{dex} \mathrm{kpc}^{-1}$.

with $\left.M_{i}^{\prime}<-2.5\right)$. At fainter magnitudes stars at the red/metalrich side of the RGB fall below the completeness limit, and the resulting MDF is artificially skewed toward the metal-poor end. We adopt four different sets of stellar evolutionary tracks from VandenBerg et al. (2006; with ages of 8 and 12 Gyr and $[\alpha / \mathrm{Fe}]$ of 0.0 and 0.3 ) and interpolate between them to derive metallicities on a star by star basis. The model grid consist of 16 finely spaced red giant tracks covering the range of metallicities from $[\mathrm{Fe} / \mathrm{H}]=-2.31$ to 0.00 in steps of approximately 0.1 dex.

We divide the data into bins of at least 200 stars and for each isochrone set determine the median metallicities in these bins. The metallicity gradient is calculated as a slope of a linear fit to the binned metallicities. Effects of using stellar tracks with different ages and/or $\alpha$-enhancements are shown in Figure 7 and Table 2 . The use of older and more $\alpha$-enhanced isochrones results in lower overall metallicities, but has practically no effect on the derived abundance gradient.

Figure 7 reveals two surprising characteristics - a strong positive metallicity gradient in the NW field and overall low metallicities in both fields. It becomes clear upon closer inspection of Figure 7 that the outermost binned data point is primarily responsible for the overall steep gradient in the NW field. The most distant radial bin covers a radial range of $\sim 3^{\prime}$; a substantial fraction of detections in these outermost few arcminutes can be attributed to background contamination (Figure 5(f)), making the number count of actual RGB stars very low. If the most distant data point is discarded, the abundance gradient becomes flat, with the slope and overall metallicity consistent with that derived for the SE field. However, the value of the slope does not change with the change of the bin size, suggesting that the positive abundance gradient in the NW field is real.

On the issue of very low metallicities, we see two possible causes for such behavior. Errors in the photometric calibration would manifest as a displacement of the RGB within the CMD, which would in turn result in false metallicity estimates. We reject this possibility, since due to the initial problems with the photometry (Section 2), photometric calibration was checked extensively and independently by Gemini personnel, and their analysis confirmed our zero-point values. Alternatively, it is possible that the component we are seeing in the outskirts of NGC 7793 is not an outer disk, but a galactic halo. However, the effective surface brightness profile we derive follows the inner disk profile (Figure 6) and it seems very unlikely that the transition from the disk to the halo occurs already at $6^{\prime}$, where we derive stellar metallicities of $[\mathrm{Fe} / \mathrm{H}] \approx-1.5$. Moreover, Radburn-Smith et al. (2011) derive metallicities in the outer disk of NGC 7793 and find low mean abundances ranging from -1.23 to -1.64 in their Fields 01 and 02 (which largely overlap with our SE field).

In order to assess the magnitude of the apparent discontinuity in the region where the inner and the outer disk metallicities overlap ( $\sim 6$ arcmin, Figure 7$)$ it is necessary to take into account (1) the $[\alpha / \mathrm{Fe}]$ ratio, which corrects for the fact that inner and outer disk abundances refer to different elements, and (2) the age-metallicity relation, which reconciles abundances in young $\mathrm{H}$ II regions with metallicities of the stars on the RGB which we have assumed to be at least 8 Gyr old. Despite numerous results pointing to a flat age-metallicity relationship in the solar neighborhood (e.g., Edvardsson et al. 1993), these works find that the relation steepens for the oldest stars. Assuming that the difference in metallicities of young $\mathrm{H}$ II regions and $8-12 \mathrm{Gyr}$ old RGB stars is $\sim 0.3-0.5$ dex and $[\alpha / \mathrm{Fe}]=0.0-0.3$, it is possible to account for the abundance difference of $0.5-0.8 \mathrm{dex}$ in the transition region in Figure 7. At the high end (i.e., $0.8 \mathrm{dex}$ ), this makes high inner disk metallicities consistent with the very low abundances we derive.

Furthermore, in deriving metallicities of $\mathrm{H}$ II regions, Edmunds \& Pagel (1984) and Webster \& Smith (1983) use strong-line abundance indicators. These have been shown to result in metallicities which are a factor of two ( 0.3 dex) higher 


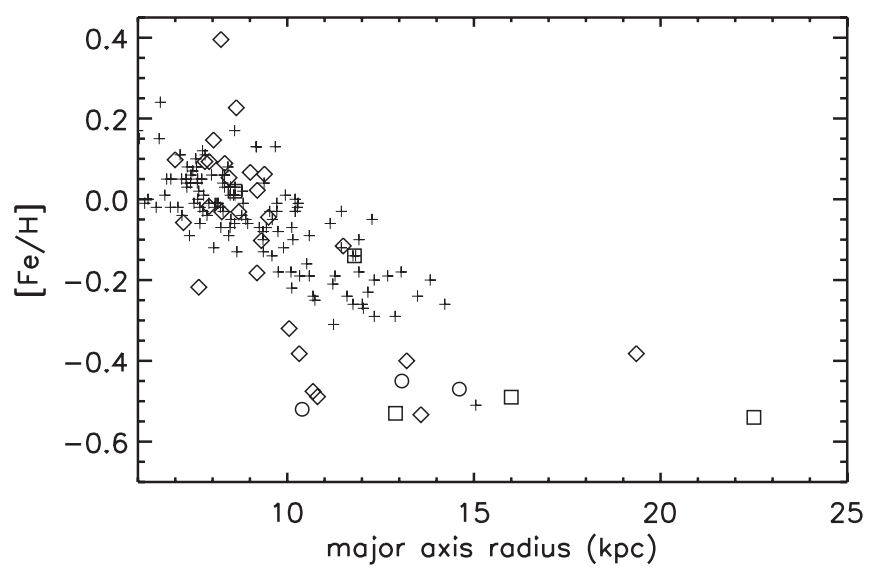

Figure 8. Metallicity gradient in the disk of the Milky Way. Plotted are data for (1) old open clusters from Twarog et al. (1997) and from Chen et al. (2003) as diamonds and Yong et al. (2005) as squares, (2) field red giants (Carney et al. 2005) as circles, and (3) Cepheids from Andrievsky et al. (2002a, 2002b), Luck et al. (2003), and Andrievsky et al. (2004) as crosses. The discrepancy is obvious between old star abundances (diamonds, squares, and crossed) and Cepheids (crosses) in the region 10-15 kpc.

than nebular ( $T_{e}$ based) or stellar abundances (Bresolin et al. 2009a) and likely enhance the difference between the inner (strong-line) and outer disk (stellar) abundances in Figure 7.

Similar abundance behavior (although of a smaller magnitude) is observed in the Milky Way. In Figure 8, we reproduce Figure 4 from Carney et al. (2005). Old open clusters are shown as diamonds (from Twarog et al. 1997; Chen et al. 2003) and squares (from Yong et al. 2005). Circles are three field red giants from Carney et al. (2005). Cepheids from the works of Andrievsky et al. (2002a, 2002b, 2002c, 2004) and Luck et al. (2003) are shown as crosses. Cepheids follow the well-defined negative gradient out to $\sim 15 \mathrm{kpc}$. On the contrary, old stars exhibit steeper gradient in the inner disk (out to $10-12 \mathrm{kpc}$ ) and a flattening in the outermost parts. In the region $10-15 \mathrm{kpc}$ there is a clear disconnect of up to 0.4 dex between the Cepheid abundances and those of old stars, similar to what we observe in NGC 7793.

\section{DISCUSSION AND CONCLUSIONS}

We can summarize the results presented thus far as follows.

1. The outer disk of NGC 7793 is primarily old with RGB stars as the dominant stellar population and a small contribution from asymptotic RGB stars.

2. After the contamination from faint background galaxies has been taken into account, number counts of RGB stars, as well as those derived using a matched catalog of all stars in the field, extend out to $\sim 10-11^{\prime}$, or $\sim 10.5-11.5 \mathrm{kpc}$.

3. The effective surface brightness profile derived from star counts traces the disk of NGC 7793 out to nine disk scale lengths and is $\sim 3 \mathrm{mag} \operatorname{arcsec}^{-2}$ deeper than the surface photometry data of Carignan (1985). Any potential break in the light profile may be associated with a specific stellar population, but we see no evidence of a truncation in old stars.

4. The metallicity gradient in the outer disk of NGC 7793 does not exhibit a negative profile, characteristic of the inner galactic regions. The value of the slope is independent of the exact set of stellar evolutionary tracks used (although the older, more $\alpha$-enhanced isochrones result in lower overall metallicities).
5. The outer disk metallicity gradient is in disagreement with the inner disk slope. The inner and outer disk abundances in the overlap region are potentially in agreement after the $[\alpha / \mathrm{Fe}]$ ratio, age-metallicity relationship, and the use of specific abundance indicators have been taken into account.

Results presented in Section 3.5 suggest that the abundance gradient derived from outer disk stellar $[\mathrm{Fe} / \mathrm{H}]$ metallicities differs in slope from the gradient calculated using $[\mathrm{O} / \mathrm{H}]$ abundances from inner disk $\mathrm{H}$ II regions (Figure 7). However, the two cannot be directly compared as the latter dataset probes recent gas abundances, while the former refers to chemical composition in stars that are at least a couple of Gyr old. In addition, given the results of Roškar et al. (2008b), which suggest that gas and stellar metallicities are decoupled and follow opposite trends, it is difficult to conclude whether our results point to an overall abundance gradient that gets shallower or steeper with time. (Models of galactic chemical evolution are successful in reproducing both trends, e.g., Molla et al. 1997; Boissier \& Prantzos 1999; Portinari \& Chiosi 1999; Tosi 1988; Chiappini et al. 2001). However, our results support the scenario presented by Roškar et al. (2008b) in which gas abundances become steeper with time (this is consistent with relatively steep inner disk slope in NGC 7793) and the stellar abundance gradient in old stars is shallower than that in young stars.

Although the stellar metallicity is the primary factor influencing colors of RGB stars, age-metallicity degeneracy and the assumption of single age in calculating the MDF introduce uncertainties in the derived MDF. As shown in Figure 7, the derived metallicity gradient is practically independent of adopted isochrones, assuming that age gradient over the extent of the disk is close to constant; a non-zero age gradient would result in a different metallicity profile. Negative age gradient in the outer disk of NGC 7793 would suggest that the real abundance gradient has a higher slope than derived under a constant age assumption. Stellar ages which decrease with radius are indeed consistent with the inside-out scenario for galaxy formation (Larson 1976; Matteucci \& Francois 1989; Chiappini et al. 1997; Naab \& Ostriker 2006; Muñoz-Mateos et al. 2007). In this picture, a galaxy's inner regions are built up at earlier times than outer parts, and as a result contain on average older stars than outermost regions. However, recent results from resolved stars (Barker et al. 2007; Williams et al. 2009, 2010) and surface photometry (Bakos et al. 2008) seem to suggest that positive age gradients are frequently observed in outer disks of spirals. This is supported by recent simulations of disk evolution (Roškar et al. 2008a, 2008b; Sánchez-Blázquez et al. 2009), which find that radial migrations of stars within the disk are responsible for the reversed age profile at large radii. If the same holds in NGC 7793, the true abundance gradient would be negative, flat, or mildly positive, depending on the magnitude of this effect.

There is a broad agreement that negative stellar abundance gradients, easily explained in the context of inside-out models for galaxy formation (Goetz \& Koeppen 1992; Matteucci \& Francois 1989), are a common feature of disk galaxies (Zaritsky et al. 1994; Ferguson et al. 1998; Gogarten et al. 2010). Surface density, yield, and star formation all decrease with radius, resulting in metallicity distribution that is more metal rich in central parts and decreases progressively toward the outer disk. However, abundance profiles in faint outer disks are more difficult to derive and there is no general consensus on their shape and origin. A growing body of evidence suggests that (most) spirals exhibit a flattening of their metallicity gradient in the outermost disk. Observationally, the strongest case has 
been made for the Galaxy (Andrievsky et al. 2004; Yong et al. 2006; Carraro et al. 2007; Pedicelli et al. 2009), M83 (Bresolin et al. 2009b) and M31 (Worthey et al. 2005). In the models of Roškar et al. (2008a, 2008b) and Sánchez-Blázquez et al. (2009), stellar radial mixing has been shown to be able to produce flat abundance profiles by "smoothing out" the underlying negative gradient. On the other hand, a mildly positive metallicity gradient has been observed in NGC 300 (Vlajić et al. 2009).

As mentioned earlier, a positive age gradient in the outer disk of NGC 7793 would bias our derivation of the abundance profile and a flat underlying metallicity profile would be observed as a positive gradient instead. Our positive metallicity gradient in the NW field could therefore be interpreted as a combination of a flat abundance and positive age gradient. This particular combination of age and metallicity behavior has been found to arise as a consequence of stellar migrations (Roškar et al. 2008b; Sánchez-Blázquez et al. 2009). On the other hand, it is possible that the positive metallicity gradient in NGC 7793 is real and does not reflect the effects of age-metallicity degeneracy. Minchev et al. (2011) find that the overlap of spiral and bar resonances in the disk triggers significant migration of stars and results in a positive abundance profile in the outermost regions, similar to what we observe in NGC 7793.

Alternatively, an external mechanism could be responsible for the shape of the metallicity gradient in outer disks of spirals. In NGC 7793, in particular, the origin of a particular abundance profile could be explained by the fact that the galaxy harbors a surprisingly small $\mathrm{H}$ I disk. While a great majority of spirals have more or less extended H I disks, sometimes stretching out far beyond the known optical edges, neutral hydrogen in NGC 7793 is detected only out to $\sim 11.5$ (Carignan \& Puche 1990; Walter et al. 2008), covering practically the same radial extent as our stellar photometry. (In addition, NGC 7793 exhibits a decreasing velocity curve in its outermost parts, which is highly unusual for a galaxy of its size.) The reason for a relatively modest $\mathrm{H}_{\mathrm{I}}$ disk in NGC 7793 is unclear, particularly given that the galaxy has no obvious interactions that could have potentially stripped the gas and truncated its $\mathrm{H}$ I distribution. Evidence for stripped stars in the outer disk of NGC 7793 is also lacking. However, it is possible to imagine that the upturn in the abundance gradient in the NW field is a consequence of a dispersed stream of stars that have long fallen below the detectability threshold in surface brightness, but still pollute the outer disk metallicities. Similar to stellar age, positive gradient in $[\alpha / \mathrm{Fe}]$ would — under the assumption of constant $[\alpha / \mathrm{Fe}]-$ be disguised as a positive gradient in metallicity. Accounting for the $[\alpha / \mathrm{Fe}]$ increase from zero to 0.3 over the range covered by our data would likely not result in a flat abundance gradient, but would certainly lower the slope of the gradient we derive for the NW field.

Finally, the shape of the metallicity gradient in the outer disk could be primordial, originating in specific galaxy formation processes taking place at high redshift. Cresci et al. (2010) show that rotating systems at $z \approx 3$ show positive abundance gradients. These are presumed to be generated by cold streams depositing pristine material into the centers of galaxies. In today's galaxies the early positive gradient in galactic center is reversed through processes of star formation and chemical evolution, while the signs of the early gradient remain in the outskirts of galaxies.

From the perspective of potential for star formation or radial migration in a given region of a spiral disk, it is interesting to examine the radial behavior of the Toomre $Q$ parameter. In a thin differentially rotating disk, rotation and pressure work to

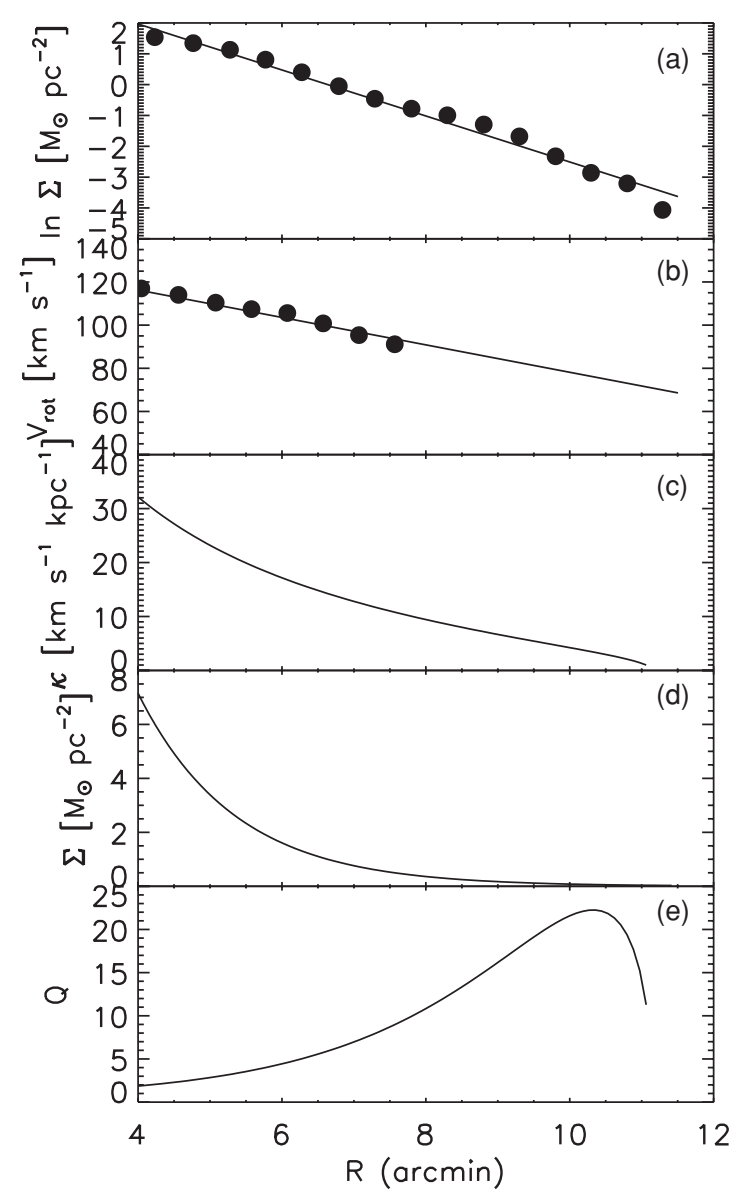

Figure 9. (a) Logarithm of surface density in the outer disk of NGC 7793. Filled circles are data from Puche et al. (1990); the solid line is a linear fit to these data points. (b) Same as (a), but for rotational velocity. (c) Epicyclic frequency derived from the velocity curve in (b). (d) Surface density. (e) Toomre $Q$ parameter.

stabilize the disk against axisymmetric perturbations. On the other hand, the disk is destabilized by its own surface density. The disk is considered unstable against axisymmetric modes if $Q$, given as

$$
Q(r)=\frac{\sigma(r) \kappa(r)}{14.45 \Sigma(r)},
$$

is less than unity. Here, $\sigma$ is radial velocity dispersion in $\mathrm{km} \mathrm{s}^{-1}$, $\kappa$ is epicyclic frequency in the units of $\mathrm{km} \mathrm{s}^{-1} \mathrm{kpc}^{-1}$, and $\Sigma$ is gas surface density in $M_{\odot} \mathrm{pc}^{-2}$. We employ $\mathrm{H}$ i observations of Puche et al. (1990) to estimate $\kappa(r)$ and $\Sigma(r)$. We approximate the velocity curve and $\log$ of the H I surface density beyond $4^{\prime}$ as linear functions of radius (Figures 9(a) and (b)) and use these fits to calculate $\kappa$ and $Q$. Epicyclic frequency $\kappa$ is calculated as

$$
\kappa^{2}=2\left(\frac{v^{2}}{r^{2}}+\frac{v}{r} \frac{d v}{d r}\right)
$$

and its radial distribution is shown in Figure 9(c). We estimate velocity dispersion in the outer parts of NGC 7793 from the THINGS survey (Walter et al. 2008) and adopt $\sigma(r)=6 \mathrm{~km} \mathrm{~s}^{-1}$ as a mean velocity dispersion in our observed field. Radial distribution of the $Q$ parameter is shown in Figure 9(e). While using a declining velocity dispersion (rather than constant as we do here) would result in a slower increase of $Q$, the Toomre parameter in the outer disk of NGC 7793 seems to 
be significantly above unity and as such does not support an environment in which there is an ongoing large-scale star formation. It is possible however that the azimuthally averaged $Q$ profile does not adequately describe star formation in outer disk. At very low gas densities in outskirts of spirals, star formation might have proceeded in a stochastic manner and in small clumps, rather than on global scales addressed by $Q$ as calculated here. This is supported by Galaxy Evolution Explorer results of clumpy star formation in outer disks of nearby spirals (e.g., Thilker et al. 2007). The high value of $Q$ presents a potential problem for radial migration as well. External perturbations, e.g., from a passing satellite galaxy, have been shown to be able to cause radial mixing in a Milky-Waytype galaxy (Quillen et al. 2009) and could be responsible for stellar migrations in high- $Q$ environments.

We thank the referee for detailed comments which contributed to the quality of the paper. Based on observations obtained at the Gemini Observatory, which is operated by the Association of Universities for Research in Astronomy, Inc., under a cooperative agreement with the NSF on behalf of the Gemini partnership: the National Science Foundation (United States), the Science and Technology Facilities Council (United Kingdom), the National Research Council (Canada), CONICYT (Chile), the Australian Research Council (Australia), Ministério da Ciência e Tecnologia (Brazil), and Ministerio de Ciencia, Tecnología e Innovación Productiva (Argentina).

\section{REFERENCES}

Andrievsky, S. M., Bersier, D., Kovtyukh, V. V., Luck, R. E., Maciel, W. J., Lépine, J. R. D., \& Beletsky, Y. V. 2002a, A\&A, 384, 140

Andrievsky, S. M., Kovtyukh, V. V., Luck, R. E., Lépine, J. R. D., Maciel, W. J., \& Beletsky, Y. V. 2002b, A\&A, 392, 491

Andrievsky, S. M., Luck, R. E., Martin, P., \& Lépine, J. R. D. 2004, A\&A, 413, 159

Andrievsky, S. M., et al. 2002c, A\&A, 381, 32

Bakos, J., Trujillo, I., \& Pohlen, M. 2008, ApJ, 683, L103

Barker, M. K., Sarajedini, A., Geisler, D., Harding, P., \& Schommer, R. 2007, AJ, 133,1125

Bellazzini, M. 2008, Mem. Soc. Astron. Ital., 79, 440

Bellazzini, M., Ferraro, F. R., \& Pancino, E. 2001, ApJ, 556, 635

Bland-Hawthorn, J., Vlajić, M., Freeman, K. C., \& Draine, B. T. 2005, ApJ, 629,239

Boissier, S., \& Prantzos, N. 1999, MNRAS, 307, 857

Bresolin, F., Gieren, W., Kudritzki, R., Pietrzyński, G., Urbaneja, M. A., \& Carraro, G. 2009a, ApJ, 700, 309

Bresolin, F., Ryan-Weber, E., Kennicutt, R. C., \& Goddard, Q. 2009b, ApJ, 695, 580

Carignan, C. 1985, ApJS, 58, 107

Carignan, C., \& Puche, D. 1990, AJ, 100, 394

Carney, B. W., Yong, D., Teixera de Almeida, M. L., \& Seitzer, P. 2005, AJ, 130,1111

Carraro, G., Geisler, D., Villanova, S., Frinchaboy, P. M., \& Majewski, S. R. 2007, A\&A, 476, 217

Chen, L., Hou, J. L., \& Wang, J. J. 2003, AJ, 125, 1397

Chiappini, C., Matteucci, F., \& Gratton, R. 1997, ApJ, 477, 765

Chiappini, C., Matteucci, F., \& Romano, D. 2001, ApJ, 554, 1044

Cresci, G., Mannucci, F., Maiolino, R., Marconi, A., Gnerucci, A., \& Magrini, L. 2010, Nature, 467,811

Da Costa, G. S., \& Armandroff, T. E. 1990, AJ, 100, 162

de Jong, R. S., et al. 2007, ApJ, 667, L49
Edmunds, M. G., \& Pagel, B. E. J. 1984, MNRAS, 211, 507

Edvardsson, B., Andersen, J., Gustafsson, B., Lambert, D. L., Nissen, P. E., \& Tomkin, J. 1993, A\&A, 275, 101

Ellis, S. C., \& Bland-Hawthorn, J. 2007, MNRAS, 377, 815

Ferguson, A. M. N., Gallagher, J. S., \& Wyse, R. F. G. 1998, AJ, 116, 673

Ferguson, A. M. N., Irwin, M. J., Ibata, R. A., Lewis, G. F., \& Tanvir, N. R. 2002, AJ, 124, 1452

Ferrarese, L., et al. 2000, ApJS, 128, 431

Goetz, M., \& Koeppen, J. 1992, A\&A, 262, 455

Gogarten, S. M., et al. 2010, ApJ, 712, 858

Iben, I., Jr., \& Renzini, A. 1983, ARA\&A, 21, 271

Irwin, M. J., Ferguson, A. M. N., Ibata, R. A., Lewis, G. F., \& Tanvir, N. R. 2005, ApJ, 628, L105

Karachentsev, I. D., et al. 2003, A\&A, 404, 93

Larson, R. B. 1976, MNRAS, 176, 31

Lee, M. G., Freedman, W. L., \& Madore, B. F. 1993, ApJ, 417, 553

Luck, R. E., Gieren, W. P., Andrievsky, S. M., Kovtyukh, V. V., Fouqué, P., Pont, F., \& Kienzle, F. 2003, A\&A, 401, 939

Martínez-Serrano, F. J., Serna, A., Doménech-Moral, M., \& DomínguezTenreiro, R. 2009, ApJ, 705, L133

Matteucci, F., \& Francois, P. 1989, MNRAS, 239, 885

Metcalfe, N., Shanks, T., Campos, A., McCracken, H. J., \& Fong, R. 2001, MNRAS, 323, 795

Minchev, I., \& Famaey, B. 2010, ApJ, 722, 112

Minchev, I., Famaey, B., Combes, F., Di Matteo, P., Mouhcine, M., \& Wozniak, H. 2011, A\&A, 527, 147

Molla, M., Ferrini, F., \& Diaz, A. I. 1997, ApJ, 475, 519

Mouhcine, M., Ibata, R., \& Rejkuba, M. 2010, ApJ, 714, L12

Muñoz-Mateos, J. C., Gil de Paz, A., Boissier, S., Zamorano, J., Jarrett, T., Gallego, J., \& Madore, B. F. 2007, ApJ, 658, 1006

Naab, T., \& Ostriker, J. P. 2006, MNRAS, 366, 899

Pedicelli, S., et al. 2009, A\&A, 504, 81

Portinari, L., \& Chiosi, C. 1999, A\&A, 350, 827

Pritchet, C. J., \& van den Bergh, S. 1994, AJ, 107, 1730

Puche, D., Carignan, C., \& Bosma, A. 1990, AJ, 100, 1468

Quillen, A. C., Minchev, I., Bland-Hawthorn, J., \& Haywood, M. 2009, MNRAS, 397, 1599

Radburn-Smith, D. J., et al. 2011, ApJ, submitted

Robin, A. C., Reylé, C., Derrière, S., \& Picaud, S. 2003, A\&A, 409, 523

Roškar, R., Debattista, V. P., Quinn, T. R., Stinson, G. S., \& Wadsley, J. 2008a, ApJ, 684, L79

Roškar, R., Debattista, V. P., Stinson, G. S., Quinn, T. R., Kaufmann, T., \& Wadsley, J. 2008b, ApJ, 675, L65

Sakai, S., Madore, B. F., \& Freedman, W. L. 1996, ApJ, 461, 713

Salaris, M., \& Cassisi, S. 1997, MNRAS, 289, 406

Sánchez-Blázquez, P., Courty, S., Gibson, B. K., \& Brook, C. B. 2009, MNRAS, 398, 591

Schönrich, R., \& Binney, J. 2009, MNRAS, 396, 203

Sellwood, J. A., \& Binney, J. J. 2002, MNRAS, 336, 785

Sharma, S., Bland-Hawthorn, J., Johnston, K., \& Binney, J. 2011, ApJ, in press

Stetson, P. B. 1987, PASP, 99, 191

Stetson, P. B. 1990, PASP, 102, 932

Thilker, D. A., et al. 2007, ApJS, 173, 538

Tosi, M. 1988, A\&A, 197, 47

Twarog, B. A., Ashman, K. M., \& Anthony-Twarog, B. J. 1997, AJ, 114, 2556

VandenBerg, D. A., Bergbusch, P. A., \& Dowler, P. D. 2006, ApJS, 162, 375

Vlajić, M., Bland-Hawthorn, J., \& Freeman, K. C. 2009, ApJ, 697, 361

Walter, F., Brinks, E., de Blok, W. J. G., Bigiel, F., Kennicutt, R. C., Thornley, M. D., \& Leroy, A. 2008, AJ, 136, 2563

Webster, B. L., \& Smith, M. G. 1983, MNRAS, 204, 743

Williams, B. F., Dalcanton, J. J., Dolphin, A. E., Holtzman, J., \& Sarajedini, A. 2009, ApJ, 695, L15

Williams, B. F., et al. 2010, ApJ, 709, 135

Worthey, G., España, A., MacArthur, L. A., \& Courteau, S. 2005, ApJ, 631, 820

Yong, D., Carney, B. W., \& Teixera de Almeida, M. L. 2005, AJ, 130, 597

Yong, D., Carney, B. W., Teixera de Almeida, M. L., \& Pohl, B. L. 2006, AJ, 131,2256

Zaritsky, D., Kennicutt, R. C., Jr., \& Huchra, J. P. 1994, ApJ, 420, 87 